\title{
summary
}

\section{Teaching critical appraisal may improve appraisal skills and knowledge but does not change reading behaviour}

Hyde C, Parkes J, Deeks J, Milne R. Systematic Review of the Effectiveness of Critical Appraisal Teaching August 2000. http://www.bham.ac.uk/arif/SysRevs/TeachCritApp.PDF

Objective To assess whether teaching critical appraisal has beneficial effects on health care workers and patients.

Data sources Ten electronic databases, the internet and references of retrieved papers. Experts and major medical education centres were also contacted.

Study selection Studies were independently assessed by three reviewers for eligibility. Data extraction and validity assessment were undertaken by one reviewer and double-checked.

Data extraction and synthesis A qualitative approach to data synthesis was used for most outcomes, due to the diversity of outcome measures, interventions, and inadequate reporting.

Results Sixteen studies met the inclusion criteria, 12 from peerreviewed journals, and four unpublished studies [one RCT, eight nonrandomised between group studies, and seven before-and-after studies]. The strongest impact of critical appraisal teaching was observed on knowledge outcomes: seven of the 12 studies showed a statistically significant positive effect. However, the size of the benefit revealed heterogeneity.
Studies showed a consistent increase in skills, with the strength of the effect remaining when self-assessed comparisons were removed. There was a mixed effect on clinicians' behaviour, with most comparisons showing a benefit.

There were four comparisons of the impact on attitudes - all were positive, but it was not possible to separate out real effects from a tendency for participants to respond in a 'desired' manner.

Conclusions There is evidence that critical appraisal teaching has positive effects on participants' attitudes, knowledge and skills. However, there are gaps in the evidence as it has an impact on decisionmaking or patient health, or on satisfaction. It is also unclear whether the benefit is substantial enough to be of practical significance. The evidence supporting all outcomes is weakened by poorly designed, executed and reported studies.

Address for correspondence: Jon Deeks, ICRF/NHS Centre for Statistics in Medicine, Institute of Health Sciences, Old Road, Headington, Oxford OX3 7LF, UK. E-mail: J.Deeks@icrf.icnet.uk

\section{Commentary}

This systematic review included studies that examined the impact of teaching critical appraisal to medical students, interns, residents, and practicing physicians in the UK, US and Canada. The authors found the studies generally to be of poor quality. Of the 16 studies included, one quarter contained major threats to validity (i.e. large loss to follow-up and inequality between groups, with no adjustment for confounding variables). In the majority of studies, those conducting the research were avid proponents of critical appraisal teaching (CAT), who possibly had a stake in the outcomes. In addition, the researchers had little or no experience in educational research, which accounts for some of the flaws in study design. None of the projects addressed either patient outcomes or satisfaction, despite the fact that the purpose of critiquing the literature is to provide better care for our patients.
Eight of the studies included in the review compared the impact from CAT on reading behaviours. Overall, there was a positive trend favouring CAT over controls. Yet, the authors point out, one of the objectives of $\mathrm{CA}$ is to teach people to read more critically, not necessarily read more. Therefore, we might hope to find a reduction in reading time, rather than an increase, for those with good CA skills. Nonetheless, sensitivity analysis revealed that the changes were not statistically significant. The impact of CAT on appraisal skills was assessed using exercises, applicationtype multiple-choice questions and self-perceived behaviours. Although 12 of the 14 comparisons favoured CAT, the size of the effect was highly variable (range of percentage change between subjects and controls was $1.4 \%$ and $42 \%$ ). Thus, the practical significance of these figures is unclear. Of the 10 comparisons favouring an impact of CAT on knowledge, seven were statistically significant. Again, the changes in test scores potentially attributable to CAT were small (2-25\%). Similar results were found from self-perceived measurements of attitudes.

Although it is heartening to find that there may be some benefit from teaching CA, it is disappointing to learn there is little highquality research to prove its impact. The authors correctly point out that consistent effort must be made in this field to increase study numbers and obtain valid results of measurable outcome, using consistent, valid instruments. As a strong believer in the value of critical appraisal, I would find comfort in knowing there is sound educational evidence to support the evaluation and integration of sound clinical evidence into our health care practices.

Debora C Matthews
Dalhousie University,
Halifax, Nova Scotia,
Canada

